\title{
ON A GENERALIZED RUBEL'S OPERATOR EQUATION
}

\author{
YU.S. LINCHUK
}

Received 02 February, 2015

\begin{abstract}
We describe all pairs of linear operators that act in spaces of analytic functions in domains and satisfy the generalized Rubel's operator equation.

2010 Mathematics Subject Classification: 47B38; 47A62

Keywords: space of analytic functions, Rubel's equation, operator equation
\end{abstract}

\section{INTRODUCTION}

Let $G$ be an arbitrary domain of the complex plane. Let $\mathscr{H}(G)$ denote the space of all analytic functions in $G$ equipped with the topology of compact convergence [6].

In [14], L.A. Rubel posed and solved the problem of finding all pairs of linear continuous functionals $L$ and $M$ on the space $\mathscr{H}(G)$ that satisfy the relation

$$
L(f g)=L(f) M(g)+L(g) M(f)
$$

for arbitrary functions $f$ and $g$ of $\mathscr{H}(G)$. Later, Nandakumar [10] and Zalcman [15] solved Rubel's problem in the class of linear functionals on the space $\mathscr{H}(G)$ in different ways. Further investigations related to the description of pairs of linear functionals on the space $\mathscr{H}(G)$ that satisfy similar relations were carried out by Nandakumar and Kannappan in $[5,13]$. These results and their generalizations were systematized by Kannappan in [4]. Further studies in this regard has been devoted to some operator modifications of (1.1) in classes of operators acting in $\mathscr{H}(G)[1,2,11]$.

In the light of the above-mentioned results there naturally arises the problem of finding all pairs of linear operators $A, B$ on the space $\mathscr{H}(G)$ such that

$$
(A(f g))(z)=(A f)(z)(B g)(z)+(A g)(z)(B f)(z)
$$

for arbitrary functions $f$ and $g$ of $\mathscr{H}(G)$ and for $z \in G$. In [3] this problem was solved only for some special classes of linear operators on $\mathscr{H}(G)$. In [7] Rubel's operator equation (1.2) was completely solved in the class of linear operators that act in spaces of analytic functions in domains. Note that all solutions of Rubel's equation in the class of linear continuous operators that act in spaces of analytic functions in arbitrary simply connected domains were described in [8]. The purpose 
of this paper is to determine all pairs of linear operators $A$ and $B$ on $\mathscr{H}(G)$ satisfying the generalized Rubel's operator equation

$$
(A(f g))(z)=\alpha(z)(A f)(z)(B g)(z)+\beta(z)(A g)(z)(B f)(z)
$$

for arbitrary functions $f$ and $g$ of $\mathscr{H}(G)$ for $z \in G$; here $\alpha, \beta$ are some fixed functions of $\mathscr{H}(G)$.

Note that in the case $B=0$ we have $A=0$. If $A=0$ then (1.3) implies that $B$ is an arbitrary linear operator on the space $\mathscr{H}(G)$. Obviously, we get in these cases that the set of solutions of (1.3) in the class of linear operators on $\mathscr{H}(G)$ coincides with the set of such operators: $A=0, B$, where $B$ is an arbitrary linear operator on the space $\mathscr{H}(G)$. We will assume that $A \neq 0$ and $B \neq 0$. We will call the pairs of such operators nontrivial solutions of (1.3).

The method of solving of equation (1.3) depends strongly on the relation between functions $\alpha$ and $\beta$. To solve our problem we will need auxiliary facts and they are also of independent interest.

\section{AuXiliary RESUlts}

Lemma 1. In order that the linear operators $A$ and $B$ on $\mathscr{H}(G)$ satisfy the equality

$$
A f B g=A g B f
$$

for any $f, g \in \mathscr{H}(G)$ it is necessary and sufficient that

$$
A f=a C f, B f=b C f,
$$

where $a, b$ are functions of $\mathscr{H}(G), C$ is a linear operator on $\mathcal{H}(G)$.

Proof. Necessity. Suppose that linear operators $A$ and $B$ on $\mathscr{H}(G)$ satisfy (2.1). If $A=0$ or $B=0$ then $A$ and $B$ are of the form (2.2). Therefore, we assume that $A \neq 0$ and $B \neq 0$.

Since $B \neq 0$, there exists $g_{0} \in \mathcal{H}(G)$ such that $\left(B g_{0}\right)(z) \not \equiv 0$ in $G$. Let $A g_{0}=a_{0}$, $B g_{0}=b_{0}$. Substituting $g=g_{0}$ in (2.1) we get

$$
b_{0}(z)(A f)(z)=a_{0}(z)(B f)(z)
$$

for any $f \in \mathscr{H}(G), z \in G$. Since $b_{0}(z) \not \equiv 0$ in $G$ and $A \neq 0$, (2.3) implies that $a_{0}(z) \not \equiv 0$ in $G$.

Let $S=\left\{z \in G: a_{0}(z) b_{0}(z)=0\right\}$. Then $S$ is an at most countable set. If $z_{0} \in G$ and $a_{0}\left(z_{0}\right)=0$ then by $k\left(z_{0}\right)$ we denote the multiplicity of the zero $z_{0}$ of $a_{0}(z)$. Similarly, if $z_{0} \in G$ and $b_{0}\left(z_{0}\right)=0$, then let $l\left(z_{0}\right)$ stand for the multiplicity of the zero $z_{0}$ of $b_{0}(z)$. For any $z_{0} \in S$ we put

$$
m\left(z_{0}\right)=\left\{\begin{array}{l}
k\left(z_{0}\right), \text { if } a_{0}\left(z_{0}\right)=0 \text { and } b_{0}\left(z_{0}\right) \neq 0 \\
l\left(z_{0}\right), \text { if } a_{0}\left(z_{0}\right) \neq 0 \text { and } b_{0}\left(z_{0}\right)=0 \\
\max \left\{k\left(z_{0}\right), l\left(z_{0}\right)\right\}, \text { if } a_{0}\left(z_{0}\right)=b_{0}\left(z_{0}\right)=0 .
\end{array}\right.
$$


Let $d(z)$ be a function of $\mathscr{H}(G)$ such that the zero set of $d$ coincides with $S$ and herewith the multiplicity of the zero $z_{0} \in S$ of $d$ is equal to $m\left(z_{0}\right)$. The existence of such a $d$ follows from the generalized Weierstrass theorem (see. [9], p. 301). If $z \in G \backslash S$ then equality (2.3) implies that

$$
\frac{b_{0}(z)}{d(z)}(A f)(z)=\frac{a_{0}(z)}{d(z)}(B f)(z)
$$

for any $f \in \mathscr{H}(G)$. Using (2.4) the definition of $d(z)$ implies that for any $z_{0} \in S$ the following equality holds

$$
\lim _{z \rightarrow z_{0}}\left(\frac{b_{0}(z)}{d(z)}(A f)(z)\right)=\lim _{z \rightarrow z_{0}}\left(\frac{a_{0}(z)}{d(z)}(B f)(z)\right)
$$

for an arbitrary $f \in \mathscr{H}(G)$ and notice that the above limits are finite. Therefore, each point of $S$ is a removable singularity of the functions $\frac{b_{0}(z)}{d(z)}(A f)(z)$ and $\frac{a_{0}(z)}{d(z)}(B f)(z)$. For any $f \in \mathscr{H}(G)$ we put

$$
(C f)(z)=\left\{\begin{array}{l}
\frac{b_{0}(z)}{d(z)}(A f)(z) \text { if } z \in G \backslash S ; \\
\lim _{t \rightarrow z}\left(\frac{b_{0}(t)}{d(t)}(A f)(t)\right), \text { if } z \in S .
\end{array}\right.
$$

It follows from the preceding arguments that $C$ acts in the space $\mathscr{H}(G)$. The linearity of $C$ is obvious. Let $a(z)=\frac{d(z)}{b_{0}(z)}, b(z)=\frac{d(z)}{a_{0}(z)}$. The functions $a(z)$ and $b(z)$ are analytic on the set $G \backslash S$ and each point of $S$ is a removable singularity of these functions. Therefore, $a(z)$ and $b(z)$ are analytic in $G$. The definition of $C$ implies that

$$
\begin{aligned}
& (A f)(z)=a(z)(C f)(z), \\
& (B f)(z)=b(z)(C f)(z)
\end{aligned}
$$

for any $f \in \mathscr{H}(G), z \in G \backslash S$.

Since $C$ acts in $\mathscr{H}(G)$ and $a, b \in \mathscr{H}(G)$, the equalities (2.5) and (2.6) hold for all $z \in G$. Thus, $A$ and $B$ are of the form (2.2). The necessity part is proved. The sufficiency of conditions of Lemma is obvious.

\section{THE MAIN RESULT}

We begin with the case when $\alpha(z) \not \equiv \beta(z)$ in $G$.

Theorem 1. Let $G$ be an arbitrary domain of the complex plane. Let $\alpha, \beta$ be arbitrary functions of $\mathscr{H}(G)$ and $\alpha(z) \not \equiv \beta(z)$ in $G$. In order that (1.3) has a nontrivial solution in the class of linear operators on $\mathcal{H}(G)$ it is necessary and sufficient that the following condition holds:

$$
\alpha(z)+\beta(z) \neq 0, \forall z \in G .
$$

Under condition (3.1) a pair of linear operators $A, B$ of $\mathscr{H}(G)$ satisfies (1.3) if and only if $A, B$ are defined by one of the following conditions: 
1) $A=0, B$ is an arbitrary linear operator on the space $\mathscr{H}(G)$;

2) $A f=\frac{a}{(\alpha+\beta) b}(f \circ \psi), B f=\frac{1}{\alpha+\beta}(f \circ \psi)$, where $a, b, \psi \in \mathscr{H}(G), b(z) \neq 0$ if $z \in G, \psi(G) \subset G$.

Proof. Assume that a pair of non-zero linear operators $A, B$ of $\mathscr{H}(G)$ satisfy relation (1.3). Interchanging $f$ and $g$ in (1.3) we get

$$
(A(f g))(z)=\alpha(z)(A g)(z)(B f)(z)+\beta(z)(A f)(z)(B g)(z)
$$

for any $f, g \in \mathscr{H}(G)$. Equalities (1.3) and (3.2) imply that

$$
(\alpha(z)-\beta(z))((A f)(z)(B g)(z)-(A g)(z)(B f)(z))=0
$$

for all $f, g \in \mathscr{H}(G), z \in G$. Since $\alpha(z) \not \equiv \beta(z)$ in $G$, (3.3) implies that the operators $A, B$ satisfy (2.1). By Lemma 1 , these operators are of the form (2.2), where $a, b$ are some functions of $\mathscr{H}(G), C$ is a linear operator on $\mathscr{H}(G)$.

Using (2.2) and (1.3) we get

$$
a(z)(C(f g))(z)=(\alpha(z)+\beta(z)) a(z) b(z)(C f)(z)(C g)(z) .
$$

Since $A \neq 0$ and $B \neq 0, a(z) \not \equiv 0, b(z) \not \equiv 0$ in $G$ and $C \neq 0$. Therefore,

$$
(C(f g))(z)=(\alpha(z)+\beta(z)) b(z)(C f)(z)(C g)(z)
$$

for all $f, g \in \mathscr{H}(G), z \in G$. Since $C \neq 0, \alpha(z)+\beta(z) \not \equiv 0$ on $G$. Using (3.4) we obtain that $\left(C_{1} f\right)(z)=(\alpha(z)+\beta(z)) b(z)(C f)(z)$ is a multiplicative operator on $\mathscr{H}(G)$ and $C_{1} \neq 0$. Therefore, there exists a function $\psi \in \mathscr{H}(G)$ such that $\psi(G) \subset$ $G$ and $C_{1} f=f \circ \psi$ [12]. Thus, $(\alpha(z)+\beta(z)) b(z)(C f)(z)=(f \circ \psi)(z)$ for all $f \in \mathscr{H}(G), z \in G$. Setting in the above equality $f \equiv 1$, we get that (3.1) holds and $b(z) \neq 0$ for all $z \in G$. We have that $C$ is of the following form:

$$
C f=\frac{1}{(\alpha+\beta) b}(f \circ \psi) \text {. }
$$

Substituting (3.5) in (2.2)we get that the operators $A$ and $B$ are of the form 2). The necessity part is proved.

By a direct calculation we obtain that the pair of operators $A$ and $B$ that is defined by 1) or 2), satisfies the generalized Rubel's operator equation (1.3).

Let us now assume that $\alpha(z) \equiv \beta(z)$ in $G$. Consider the operator equation

$$
(A(f g))(z)=\alpha(z)((A f)(z)(B g)(z)+(A g)(z)(B f)(z)) .
$$

Theorem 2. Let $G$ be an arbitrary domain of the complex plane. Let $\alpha$ be an arbitrary function of $\mathscr{H}(G)$. In order that operator equation (3.6) has a non-trivial solution in the class of linear operators on $\mathscr{H}(G)$ it is necessary and sufficient that the following equality holds:

$$
\alpha(z) \neq 0, \forall z \in G
$$


Under condition (3.7) a pair of linear operators $A, B$ of $\mathscr{H}(G)$ satisfies (3.6) if and only if either $A=0$ and $B$ is an arbitrary linear operator on the space $\mathscr{H}(G)$, or the pair of operators $A, B$ is defined by one of the following conditions:

1) $A(f)=\varphi \cdot(f \circ \psi) ; B(f)=\frac{1}{2 \alpha}(f \circ \psi)$, where $\varphi, \psi \in \mathscr{H}(G)$, with $\psi(G) \subset G$;

2) $A(f)=\varphi \cdot\left(f^{\prime} \circ u\right), B(f)=\frac{1}{\alpha}(f \circ u)$, where $\varphi, u \in \mathscr{H}(G)$, with $u(G) \subset G$;

$3)$ the operators $A$ and $B$ are defined by the following formulas

$$
\begin{gathered}
(A f)(z)=\left\{\begin{array}{l}
\varphi(z) \frac{f(u(z)+\sqrt{v(z)})-f(u(z)-\sqrt{v(z)})}{2 \sqrt{v(z)}}, \text { if } z \in V, \\
\varphi(z) f^{\prime}(u(z)), \text { if } z \in G \backslash V ;
\end{array}\right. \\
(B f)(z)=\left\{\begin{array}{l}
\frac{f(u(z)+\sqrt{v(z)})+f(u(z)-\sqrt{v(z)})}{2 \alpha(z)}, \text { if } z \in V, \\
\frac{1}{\alpha} f(u(z)), \text { if } z \in G \backslash V,
\end{array}\right.
\end{gathered}
$$

where $\varphi, u, v \in \mathscr{H}(G)$ and $v \not \equiv 0$, the set $G \backslash V$ coincides with the zero set of the function $v(z)$; the functions $u(z)$ and $v(z)$ are such that the functions $\chi_{1}(z)=u(z)+$ $\sqrt{v(z)}$ and $\chi_{2}(z)=u(z)-\sqrt{v(z)}$ satisfy the following conditions: $\chi_{1}(G) \subset G$, $\chi_{2}(G) \subset G$.

Proof. Assume that the nonzero linear operators $A$ and $B$ of $\mathscr{H}(G)$ satisfy (3.6). Let us show that (3.7) holds. To obtain a contradiction we suppose that there exists a point $z_{0} \in G$, such that $\alpha\left(z_{0}\right)=0$. Substituting $g \equiv 1$ in (3.6) we obtain that $(A f)\left(z_{0}\right)=0$ holds for any $f \in \mathscr{H}(G)$. Let $n(f)$ stands for the multiplicity of the zero $z_{0}$ of the function $A f$ (in the case when $(A f)(z) \equiv 0$ in $G$ we put $n(f)=+\infty$ ). Let $n_{0}=\min \{n(f): f \in \mathscr{H}(G)\}$. By the assumption the operator $A$ is nonzero. Then for an arbitrary $f \in \mathscr{H}(G)$ the point $z_{0}$ is a removable singularity for the function $\frac{(A f)(z)}{\left(z-z_{0}\right)^{n_{0}}}$. Define $\left(A_{1} f\right)(z)=\frac{(A f)(z)}{\left(z-z_{0}\right)^{n} 0}$ if $z \neq z_{0}$. Then $A_{1}$ can be extended to an operator which linearly acts in $\mathscr{H}(G)$. The definition of $n_{0}$ implies that there exists the function $f_{0} \in \mathscr{H}(G)$ such that $\left(A_{1} f_{0}\right)\left(z_{0}\right) \neq 0$. For any function $f \in \mathscr{H}(G)$ the following equality holds:

$$
(A f)(z)=\left(z-z_{0}\right)^{n}\left(A_{1} f\right)(z) .
$$

Substituting (3.8) in (3.6) we obtain that

$$
\left(A_{1}(f g)\right)(z)=\alpha(z)\left(\left(A_{1} f\right)(z)(B g)(z)+\left(A_{1} g\right)(z)(B f)(z)\right)
$$

for all $f, g \in \mathscr{H}(G), z \in G$. Setting $g \equiv 1, f=f_{0}$ and $z=z_{0}$ in (3.9) we get a contradiction. Thus, (3.7) holds.

Under condition (3.7) a pair of linear operators $A, B$ on $\mathscr{H}(G)$ satisfies (3.6) if and only if the pair of operators $A, \alpha B$ satisfy Rubel's equation (1.2). Therefore, the assertion of the theorem follows from the main theorem of [7].

\section{REFERENCES}

[1] J. Becker, "A note on derivations of algebras of analytic functions," J. Reine Angew. Math., vol. 297, pp. 211-213, 1978, doi: 10.1515/crll.1978.297.211. 
[2] R. B. Burckel and S. Saeki, "Additive mappings on rings of holomorphic functions," Proc. Amer. Math. Soc., vol. 89, no. 1, pp. 79-85, 1983, doi: 10.2307/2045068.

[3] A. K. Gaur and N. R. Nandakumar, "Derivation pairs of operators on algebras of analytic functions," Functional analysis: selected topics, Narosa, New Delhi, pp. 104-110, 1998.

[4] P. Kannappan, Functional Equations and Inequalities with Applications, ser. Springer Monographs in Mathematics. New York: Springer, 2009.

[5] P. Kannappan and N. R. Nandakumar, "On a cosine functional equation for operators on the algebra of analytic functions in a domain," Aeq. Math., vol. 61, no. 3, pp. 233-238, 2001, doi: 10.1007/s000100050175.

[6] G. Köthe, "Dualität in der funktionentheorie," J. R eine Angew. Math., vol. 191, pp. 30-49, 1953.

[7] Y. S. Linchuk, "On Rubel's problem in the class of linear operators on the space of analytic functions," Complex Anal. Oper. Theory., vol. 63, no. 12, pp. 1945-1952, 2012, doi: 10.1007/s11253012-0623-3.

[8] Y. S. Linchuk, “Operator generalization of one Rubel's result," Ukr. Math. J., vol. 8, no. 8, pp. 1741-1745, 2014, doi: 10.1007/s11785-014-0366-2.

[9] A. I. Markushevich, Theory of analytic functions, 2nd ed. Moscow: Nauka, 1968, vol. II.

[10] N. R. Nandakumar, "A note on derivation pairs," Proc. Amer.Math.Soc., vol. 21, pp. 535-539, 1969, doi: 10.1090/s0002-9939-1969-0240606-9.

[11] N. R. Nandakumar, "An application of Nienhuys-Thiemann s theorem to ring derivations on h(g)," Indag. Math., vol. 50, no. 2, pp. 199-203, 1988, doi: 10.1016/s1385-7258(88)80027-1.

[12] N. R. Nandakumar, “Ring homomorphisms on h(g)," Int. J. Math. Sci., vol. 13, no. 2, pp. 393-396, 1990.

[13] N. R. Nandakumar, "A note on the functional equation $m(f g)=m(f) m(g)+l(f) l(g)$ on $h(g)$," Rend. Sem. Fac. Sci. Univ. Cagliari., vol. 68, pp. 13-17, 1998.

[14] L. A. Rubel, "Derivation pairs on the holomorphic functions," Funkcial. Ekvac., vol. 10, pp. 225 227, 1967.

[15] L. Zalcman, "Derivation pairs on algebras of analytic functions," J. Func. Anal., vol. 5, no. 3, pp. 329-333, 1970, doi: 10.1016/0022-1236(70)90012-1.

\section{Author's address}

\section{Yu.S. Linchuk}

Chernivtsi National University, Department of Mathematical Analysis, Kotsjubyns'koho 2, 58012

Chernivtsi, Ukraine

E-mail address: yustlin@gmail.com 